Angélica Cecília

TATARUNAS ${ }^{1}$

Julia Maria MATERA ${ }^{1}$

Renata Moris Domenico

OLIVEIRA ${ }^{1}$

Renata Ferri MACCHIONE ${ }^{1}$

Milena BRUGNARO'

Correspondência para:

Rua Orlando Marques de Paiva, 87

Butantã-Cidade Universitária

05508900 - São Paulo, SP

e-mail:angelvet@usp.br

Recebido para publicação: 12/12/2004 Aprovado para publicação: 27/03/2007

\title{
Estudo retrospectivo do tratamento da ruptura de ligamento cruzado cranial no cão por técnica cirúrgica extra-articular
}

1 - Departamento de Cirurgia, Faculdade de Medicina Veterinária e

Zootecnia, Universidade de São Paulo, São Paulo-SP

\begin{abstract}
Resumo
As técnicas extra-capsulares têm sido amplamente utilizadas para a reparação da ruptura do ligamento cruzado cranial no cão (RLCC); e, a sutura fabelo tibial tem se tornado uma das mais populares. A presente pesquisa teve por finalidade avaliar a evolução clínica de pelo menos 4 meses de pós-operatório de 19 cães (20 articulações) portadores de RLCC e submetidos a técnica acima descrita. Realizouse entrevista com o proprietário e exame clínico consistindo de avaliação do escore de claudicação, teste de gaveta e mensuração do diâmetro da coxa, volume e ângulo da articulação do joelho. De acordo com os proprietários $90 \%$ dos animais apresentaram recuperação boa ou ótima após o tratamento cirúrgico, variando de ausência de claudicação com claudicação esporádica. A avaliação clínica denotou resultado favorável no que tange ao teste de gaveta e escore de claudicação. As mensurações objetivas, contudo, não corroboraram na mesma proporção com os resultados clínicos.
\end{abstract}

Palavras-chave: Articulação. Joelho. Cães. Cirurgia.

\section{Introdução e Revisão de Literatura}

A ruptura de ligamento cruzado cranial (RLCC) é uma das afecções articulares de maior prevalência no cão ${ }^{1,2,3}$ e o tratamento cirúrgico é considerado de eleição ${ }^{4,5,6,7}$. Dentre as técnicas cirúrgicas descritas para a reparação desta afecção ${ }^{8,9,10}$, a ancoragem de um fio de sutura ao redor da fabela lateral e em um túnel na crista da tíbia é uma das mais populares entre os veterinários e o objetivo consiste em abolir ou restringir o movimento de gaveta cranial a um valor menor ou igual a $3 \mathrm{~mm}^{11-15}$. São citados vários materiais de sutura como sendo passíveis de serem utilizados com esta finalidade (náilon, polipropileno, poliéster, polidioxanona, poligliconato, caprolactam e fio de aço), e os autores têm dado preferência a fio monofilamentar e não absorvível ${ }^{11,16-23}$.

Foram realizados estudos biomecânicos da técnica extra-articular de sutura fabelo tibial pela análise do centro instantâneo do movimento. Enquanto Arnoczky e Marshall ${ }^{24}$ afirmam que as articulações apresentaram movimento anormal das superfícies articulares, predispondo a lesões de menisco e cartilagem, Prostredny et al. ${ }^{21} \mathrm{e}$, mais recentemente, Selmi et al. ${ }^{22}$, obtiveram biomecânica articular normal com o uso dos fios de polibutester, poliéster torcido e polipropileno.

Dulish ${ }^{25}$ relatou edema excessivo com ou sem fístula em $21 \%$ de cães submetidos à técnica de imbricação do retináculo modificada $^{13} \mathrm{com}$ fio multifilamentar e associa a ele contaminação por Staphylococcus aureus e Micrococcus spp, reação imunomediada e/ou reação alérgica ao material de sutura. Em outro estudo realizado pelo mesmo autor, observou-se $18 \%$ de complicações relacionadas com o material de sutura e a conclusão é de que as reações resultaram de suturas contaminadas ${ }^{26}$.

Vários estudos in vitro discutindo material de sutura, modo de esterilização, tipo de nó, utilização de grampos especiais em substituição à colocação de nós e também o efeito do pinçamento do fio de 
sutura durante a confecção da primeira laçada do nó têm sido realizados ${ }^{11,16,19,20,27}$, porém a literatura carece de estudos de avaliação clínica com a aplicação da técnica.

Em entrevista com 175 cirurgiões, Korvick, Johnson e Schaeffer ${ }^{17}$ concluíram que os fios de maior preferência para a realização de técnica extracapsular foram o náilon e o polipropileno. A presente pesquisa teve por finalidade avaliar a evolução clínica de pelo menos quatro meses de pósoperatório de cães submetidos a reparação cirúrgica de RLCC pela técnica de sutura fabelo tibial utilizando os fios de náilon monofilamento ou o fio de polipropileno.

\section{Material e Método}

Foram utilizados 19 cães (20 articulações) com diagnóstico de RLCC, atendidos no Serviço de Cirurgia de Pequenos Animais do Hospital Veterinário da Faculdade de Medicina Veterinária e Zootecnia da Universidade de São Paulo. Durante o exame clínico avaliou-se a presença de instabilidade crânio caudal da tíbia em relação aos côndilos do fêmur (teste de gaveta) com a articulação em posição anatômica, flexão e extensão; e o escore de claudicação (Quadro 1). Neste momento foi realizado estudo radiográfico, classificandose a doença articular degenerativa quando presente segundo escala de Moreau et al. ${ }^{28}$.

Inicialmente realizou-se inspeção da articulação por artroscopia segundo técnica de Whitney ${ }^{29}$, confirmando-se desta forma a presença de RLCC e dando-se especial ênfase ao exame dos meniscos. Na seqüência fez-se abordagem parapatelar lateral da articulação segundo técnica de Piermattei e $\operatorname{Johnson}^{30}$, remoção dos resquícios de ligamento cruzado cranial rompido e meniscectomia parcial ou total se necessário. A síntese da porção sinovial da cápsula articular foi realizada com Poliglecaprone 30 (Monocryl $^{\mathbb{B}}$ - Ethicon - Johnson \& Johnson) em pontos não transfixantes e realizou-se a imbricação lateral do retináculo ${ }^{7}$ em dois planos com fio de náilon 3-0 (Mononylon $^{\circledR}$ - Ethicon - Johnson \& Johnson).
Para a sutura fabelo tibial lateral utilizou-se um a dois ${ }^{7}$ fios de náilon 0 Mononylon $^{\circledR}$ - Ethicon - Johnson \& Johnson) ou polipropileno 2 (Prolene ${ }^{\circledR}$ Ethicon - Johnson \& Johnson), dependendo do peso do animal. Este foi passado atrás da fabela lateral com auxílio de agulha de Martin e através de um túnel confeccionado na porção proximal da tuberosidade da tíbia. Utilizou-se agulha hipodérmica 40x12 como guia para passar o fio no túnel tibial e sobre o ligamento patelar, para posteriormente unir as duas extremidades próximas à tuberosidade da tíbia com confecção de cinco nós quadrados ${ }^{11}$. O deslocamento cranial da tíbia em relação ao fêmur foi reduzido e foi feita a rotação externa da tíbia anterior à confecção do nó. Posteriormente foi realizado síntese da fáscia superficial com pontos simples separados utilizando fio de poliglecaprone4-0 (Monocryl $^{\circledR}$ - Ethicon Johnson \& Johnson) e sutura intradérmica da pele com fio de náilon 4-0 (Mononylon ${ }^{\circledR}$ - Ethicon - Johnson \& Johnson).

A avaliação dos 19 cães (20 articulações) consistiu em um retorno tardio ao Hospital Veterinário visando a análise do proprietário e do médico veterinário sobre o resultado final obtido do procedimento cirúrgico realizado.

Solicitou-se aos proprietários que classificassem o resultado final do tratamento como ótimo, bom, regular ou ruim, segundo a função do membro durante a locomoção (ausência de claudicação, claudicação esporádica e claudicação constante). $\mathrm{O}$ intervalo de tempo entre a realização da intervenção cirúrgica e a melhora total do membro operado também lhes foi questionado.

A avaliação pelo médico veterinário consistiu de exame físico, escore de claudicação (Quadro 1) e teste de gaveta. Também a mensuração do ângulo da articulação do joelho, com uso de goniômetro, em flexão e extensão máximas sem promover desconforto para o animal; a medida do diâmetro da coxa, com o auxílio de fita métrica; e do volume articular, com paquímetro (da borda medial à borda lateral 


\begin{tabular}{|cc|}
\hline Escore & Significado \\
\hline 0 & Ausência de claudicação \\
\hline 1 & Leve anormalidade do andar. Claudicação leve \\
\hline 2 & Claudicação evidente \\
\hline 3 & Óbvia anormalidade do andar. Impotência funcional em repouso. Apoio em movimento \\
\hline 4 & Anormalidade grave do andar. Impotência funcional intermitente durante locomoção \\
\hline 5 & Impotência funcional \\
\hline
\end{tabular}

Quadro 1 - Escore de claudicação. São Paulo, 2004

da articulação). Estes dados foram tomados no membro operado e contralateral.

\section{Resultados}

O intervalo de tempo entre a realização da intervenção cirúrgica e a reavaliação do retorno tardio variou de quatro a 29 meses, com uma média de 14 meses.

As raças, idade, sexo e peso dos animais avaliados encontram-se dispostas na tabela 2. As raças Boxer, Pit Bull e Poodle foram as mais acometidas (15,8\% cada), sendo que um animal da raça Boxer apresentou a afecção bilateral. Os cães sem raça definida compreenderam 15,78\%. Catorze animais foram fêmeas e cinco machos; a idade variou de um a 10anos ( $\mathrm{x}=$ $4,7$ anos) e o peso de 4,8 a $38 \mathrm{~kg}$ ( $x=21,6 \mathrm{~kg})$.

Um dos cães apresentou comprometimento bilateral das articulações do joelho em intervalo de um ano. Entre os demais, 50\% teve a articulação direita e 50\% a esquerda acometida.

Anteriormente ao ato cirúrgico todos os animais apresentaram teste de gaveta cranial positivo e o escore de claudicação foi $3 \mathrm{em}$ cinco membros, 4 em 13 e 5 em dois $(x=3,8)$. O escore radiográfico préoperatório denotou graduação $0 \mathrm{em}$ seis articulações, 1 em 10 e 2 em quatro $(x=0,9)$.

Durante o exame artroscópico foram detectadas $17(85 \%)$ rupturas totais e três $(15 \%)$ rupturas parciais do ligamento cruzado cranial, laceração em um $(5 \%)$ menisco lateral e lesão em oito (40\%) meniscos mediais. Foram realizadas oito meniscectomias mediais parciais e uma exérese total de menisco lateral total (Tabela 1).
A relação dos fios utilizados para a sutura fabelo tibial em cada articulação encontra-se na tabela 2 .

\section{Avaliação realizada pelo proprietário}

Os resultados referentes à avaliação do proprietário e tempo de recuperação do membro operado encontram-se na tabela 3.

O retorno ótimo à função foi relatado em $13(65 \%)$ membros, bom em seis (30\%) e regular em um $(5 \%)$. O tempo médio de recuperação após a cirurgia variou de $\leq 2$ meses em oito (40\%) membros, 3 a 4 meses em nove $(45 \%)$ e $>5$ meses em três $(15 \%)$.

\section{Avaliação realizada pelo médico veterinário}

Os resultados concernentes ao exame clínico encontram-se na tabela 2.

O teste de gaveta foi nulo em 18 $(90 \%)$ articulações e dois $(10 \%)$ apresentaram leve instabilidade. $\mathrm{O}$ escore de claudicação foi 0 para $12(60 \%)$ membros pélvicos e 1 para oito $(40 \%)$.

As medidas do volume articular da articulação do joelho operado e do contralateral foram subtraídas sendo o resultado entre 0 e $0,5 \mathrm{~cm}$ em 14 articulações $(77,78 \%)$ e de 0,6 a $1,1 \mathrm{~cm}$ em quatro $(22,22 \%)$. O cão portador de RLCC bilateral apresentou diferença de $0,1 \mathrm{~cm}$ entre as duas articulações do joelho operadas.

A medida do diâmetro da coxa do membro operado e contralateral foram subtraídas, sendo o resultado entre 0 e $1 \mathrm{em}$ 12 articulações $(66,7 \%)$; de 1,5 a 2 em 4 $(22,2 \%)$ e 2,5 em $2(11,1 \%)$. O cão com RLCC bilateral não apresentou diferença na medida entre as duas coxas. 
Tabela 1 - Relação dos cães portadores de RLCC operados no HOVET/FMVZ/USP, quantoà raça, sexo, idade, peso, membro acometido, escore de claudicação e do RX pré-operatórios, intervalo de tempo entre a cirurgia e a reavaliação no pós-operatório tardio, diagnóstico e material de sutura utilizado na cirurgia. São Paulo, 2004

\begin{tabular}{lcccccccccc}
\hline $\mathrm{N}$ & Raça & $\begin{array}{c}\text { Sexo } \\
\text { (anos) }\end{array}$ & Idade & $\begin{array}{c}\text { Peso } \\
(\mathrm{Kg})\end{array}$ & $\begin{array}{c}\text { Membro } \\
\text { Claudicação }\end{array}$ & $\begin{array}{c}\text { Escore } \\
\text { RX }\end{array}$ & $\begin{array}{c}\text { Escore } \\
\text { IC/RT }\end{array}$ & $\begin{array}{c}\text { T } \\
\text { Diagnóstico }\end{array}$ & $\mathrm{N}^{\mathbf{0}}$ \\
tipofio
\end{tabular}

$\mathrm{N}$ : Número da articulação, D: Direito, E: Esquerdo, TIC/RT: tempo de intervalo entre a intervenção cirúrgica e a reavaliação tardia; T: RLCC total P: RLCC parcial; T/Mm: RLCC total com lesão de menisco medial; T/Ml: RLCC total com lesão de menisco lateral; P: Fio de sutura de polipropileno $\mathrm{n}^{\circ} 2 ; \mathrm{N}$ : Fio de sutura de náilon $\mathrm{n}^{\circ} 0$

A medida do ângulo da articulação do joelho operado em flexão máxima foi maior ou igual ao contralateral em 10 $(55,6 \%)$ e menor em $8(44,4 \%)$ articulações. A medida do ângulo da articulação do joelho operado em extensão máxima foi maior ou igual ao contralateral em sete $(38,9 \%)$ e menor em $11(61,1 \%)$ articulações. A goniometria do cão com RLCC bilateral não foi considerada.

\section{Discussão}

A RLCC pode ocorrer em qualquer raça da espécie canina, porém as de grande porte são principalmente acometidas ${ }^{1,2,29}$. O grupo de cães estudados foi representado por diferentes padrões raciais, sendo que o Boxer, Poodle, Pit Bull e sem raça definida compreenderam 15,8\%.

Segundo Vasseur ${ }^{31}$, a RLCC no cão geralmente está associada com alterações degenerativas do próprio ligamento e as raças maiores apresentam o evento mais precocemente. No presente estudo 57,9\% dos animais possuía idade inferior a quatro anos e entre estes oito $(72,7 \%)$ apresentavam peso superior a $17,4 \mathrm{~kg}(\mathrm{x}=21,6)$, coincidindo com os dados acima.

Em vários estudos as fêmeas apresentam maior incidência da afecção ${ }^{2,3}$, fato aqui constatado onde o sexo feminino representou $73,7 \%$ dos cães acometidos.

Em animais submetidos a tratamento cirúrgico, tem-se observado no ligamento cruzado cranial doente, alterações tais como: fibrilação, estiramento, ruptura parcial e ruptura total, denotando um processo degenerativo seqüencial ${ }^{32}$. Foram detectadas três $(15,8 \%)$ rupturas parciais, todas em grau acentuado de deterioração, e comprometendo tanto a banda craniomedial 
Tabela 2- Relação dos cães portadores de RLCC quanto aos resultados obtidos sobre a avaliação do proprietário (resultado final e tempo de melhora total do membro operado) e do medico veterinário (teste de gaveta, score de claudicação, diâmetro da coxa do membro operado e contralateral, ângulo da articulação do joelho operado e contra lateral na flexão e na extensão). São Paulo, 2004

\begin{tabular}{|c|c|c|c|c|c|c|c|c|c|c|c|c|}
\hline \multirow{2}{*}{ N. } & \multirow{2}{*}{$\begin{array}{l}\text { Resultado } \\
\text { final }\end{array}$} & \multirow{2}{*}{$\begin{array}{c}\text { Melhora } \\
\text { (dias) }\end{array}$} & \multirow{2}{*}{ TG } & \multirow{2}{*}{$\mathrm{EC}$} & \multicolumn{2}{|c|}{ VA } & \multicolumn{2}{|c|}{$\mathrm{DC}$} & \multicolumn{2}{|c|}{ GF } & \multicolumn{2}{|c|}{ G E } \\
\hline & & & & & $\mathrm{O}$ & $\mathrm{CL}$ & $\mathrm{O}$ & CL & $\mathrm{O}$ & $\mathrm{CL}$ & $\mathrm{O}$ & $\mathrm{CL}$ \\
\hline $1 \mathrm{D}$ & Ótimo & 90 & A & 0 & 5,6 & 5,5 & 35 & 35 & 73 & 60 & 158 & 140 \\
\hline $1 \mathrm{E}$ & Ótimo & 180 & A & 0 & 5,5 & 5,6 & 35 & 35 & 60 & 73 & 140 & 158 \\
\hline 2 & Ótimo & 90 & $\mathrm{~A}$ & 0 & 5,2 & 5,0 & 43 & 45 & 80 & 60 & 144 & 150 \\
\hline 3 & Bom & 30 & A & 0 & 4,0 & 3,9 & 20,5 & 20,7 & 82 & 60 & 140 & 134 \\
\hline 4 & Bom & 90 & A & 1 & 5,8 & 6,3 & 45 & 45 & 84 & 76 & 148 & 158 \\
\hline 5 & Ótimo & 60 & A & 0 & 3,2 & 2,7 & 16,0 & 16,5 & 64 & 78 & 170 & 150 \\
\hline 6 & Ótimo & 120 & A & 0 & 4,6 & 4,5 & 32 & 32,5 & 50 & 48 & 140 & 156 \\
\hline 7 & Ótimo & 90 & A & 0 & 3,2 & 3,2 & 26 & 25,5 & 36 & 30 & 156 & 114 \\
\hline 8 & Ótimo & 90 & $\mathrm{~A}$ & 1 & 5,2 & 4,8 & 37 & 39 & 52 & 50 & 138 & 158 \\
\hline 9 & Ótimo & 180 & A & 0 & 4,7 & 4,6 & 39 & 40 & 56 & 52 & 146 & 150 \\
\hline 10 & Regular & 60 & A & 1 & 6,2 & 5,6 & 32 & 33,5 & 50 & 56 & 146 & 154 \\
\hline 11 & Bom & 210 & + & 1 & 5,2 & 5,9 & 33 & 35,5 & 96 & 102 & 112 & 142 \\
\hline 12 & Ótimo & 7 & A & 1 & 4,0 & 4,1 & 23,5 & 24 & 52 & 52 & 134 & 134 \\
\hline 13 & Bom & 150 & A & 1 & 4,5 & 4,6 & 42 & 42 & 44 & 36 & 132 & 140 \\
\hline 14 & Ótimo & 150 & A & 0 & 5,4 & 5,1 & 43 & 45 & 68 & 56 & 112 & 122 \\
\hline 15 & Ótimo & 60 & A & 0 & 3,4 & 3,5 & 17,5 & 17,4 & 52 & 30 & 122 & 104 \\
\hline 16 & Ótimo & 60 & + & 1 & 4,5 & 4,5 & 27,5 & 26,5 & 48 & 52 & 140 & 140 \\
\hline 17 & Bom & 120 & A & 0 & 6,0 & 4,9 & 36 & 38,5 & 64 & 42 & 140 & 154 \\
\hline 18 & Bom & 60 & A & 1 & 6,0 & 5,3 & 35 & 35 & 76 & 58 & 147 & 145 \\
\hline 19 & Ótimo & 90 & A & 0 & 2,0 & 2,0 & 17,5 & 18 & 56 & 56 & 160 & 146 \\
\hline
\end{tabular}

N: Número da articulação, D: Direito, E: Esquerdo, TG: Teste de Gaveta, A: Ausente, + : gaveta presente, EC: Escore de Claudicação, VA: Volume Articular, DC: Diâmetro da Coxa, G F Goniometria durante a flexão articular máxima da articulação do joelho; G E Goniometria durante a extensão articular máxima da articulação do joelho, O: membro operado, CL: membro contralateral

como a caudolateral do ligamento cruzado cranial $^{24}$.

A RLCC freqüentemente está associada com a lesão do menisco medial, sendo superior a $75 \%$ em alguns relatos ${ }^{5,32}$. Mais recentemente, sob visualização artroscópica, Ralphs e Whitney ${ }^{33}$ relataram $77 \%$ de acometimento do menisco lateral e $58 \%$ do menisco medial. Nos animais avaliados a prevalência de lesão foi de $40 \%$ para o menisco medial, sendo a maioria lesão em alça de balde e 5\% para o menisco lateral, que consistiu de laceração em grau avançado. Durante o exame artroscópico detectou-se a presença de fibrilação da porção medial de grande parte dos meniscos laterais, porém estas alterações não foram consideradas. Acredita-se que com o aumento do uso da artroscopia novas informações serão adicionadas ao estudo desta afecção e desta forma, conceitos e classificações terão de ser revistos pelos pesquisadores.

As pesquisas clínicas de cães portadores de RLCC e submetidos à reparação cirúrgica têm se baseado em entrevista com o proprietário, reavaliação clínica dos animais ${ }^{6,8}$ e, mais recentemente estudos de cinética e cinemática ${ }^{34,35}$. Seguramente o uso de placa de força e/ou a análise cinemática do movimento são formas de avaliação mais fidedignas ${ }^{34}$, porém requerem equipamento e profissionais especializados.

Neste estudo, 90\% dos proprietários consideraram o resultado final obtido entre ótimo e bom, variando entre a ausência de claudicação e a claudicação esporádica. Tal fato corrobora com os resultados obtidos por Chauvet et al. ${ }^{4}$, Coetzee e Lubee ${ }^{8}$ e Hulse et al. ${ }^{14}$ e considerados favoráveis com diferentes técnicas para a reparação da RLCC no cão.

$\mathrm{O}$ procedimento cirúrgico para a 
reparação do ligamento cruzado cranial rompido visa restabelecer a estabilidade articular e promover o retorno à função do membro ${ }^{11,12,14,15}$. Haja visto que anteriormente ao tratamento cirúrgico todos os cães deste estudo apresentavam o teste de gaveta positivo e, durante a reavaliação, este teste foi negativo em $90 \%$ das articulações, obteve-se resultado favorável no que tange a estabilidade articular na maioria dos animais. Quanto à função do membro operado, o escore pré-operatório variou de 3 a 5 (Quadro 1) e aquele obtido na avaliação tardia entre 0 e 1 (Quadro 1), coincidindo com os resultados obtidos pela avaliação do proprietário.

É sabido que nenhuma técnica operatória descrita para reparar a RLCC na espécie canina evita a evolução da doença articular degenerativa ${ }^{4,7,21} \mathrm{e}$, dentre os animais estudados, $70 \%$ das articulações apresentavam alterações radiográficas compatíveis com doença articular degenerativa no pré-operatório. Quanto a mensuração do volume articular, $77,8 \%$ dos cães apresentaram ausência ou aumento discreto do membro operado em relação ao contralateral, e $22,2 \%$ das articulações o apresentaram entre 0,6 e $1,1 \mathrm{~cm}$. Considerando a reavaliação durante o pósoperatório tardio, tal fato pode estar relacionado com o processo de remodelamento articular, haja visto que a maioria dos animais apresentava sinais radiográficos de doença articular degenerativa no momento do diagnóstico.

Em cães com RLCC, a atrofia muscular geralmente envolve os músculos quadríceps e bíceps femoral ${ }^{6}$. Aferiu-se o diâmetro da coxa e, considerando-se o membro contralateral como medida padrão para o restabelecimento da massa muscular, obteve-se diâmetro próximo ou igual ao contralateral em $61,1 \%$.

Moore e Read ${ }^{6}$ relataram diminuição significativa da amplitude articular durante a flexão e a extensão após a realização de sutura fabelo tibial para a reparação da RLCC e atribuem esta à fibrose periarticular conseqüente ao fio implantado, incisão cirúrgica ou ainda a imbricação da fáscia lateral. Em nosso estudo, tal fato foi constatado em $44,4 \%$ dos animais durante a flexão e em $61,1 \%$ durante a extensão, concordando com os dados acima.

Como complicação do uso desta técnica, Dulish ${ }^{25}$ relata edema excessivo com ou sem fístula e posteriormente o associa com contaminação bacteriana ${ }^{26}$. Com aproximadamente 30 dias de pós-operatório observou-se nos animais $\mathrm{n}^{\circ} 11$ e 17 aumento de volume indolor a dígito pressão, circunscrito, flutuante, com cerca de 0,5 a $1,0 \mathrm{~cm}$ de diâmetro junto a porção medial da tuberosidade da tíbia, os quais apresentaram regressão com o uso de compressa quente e não houve recidiva até o momento do exame do pós-operatório tardio. Apesar de não ter sido realizado punção local e encaminhado material para cultura de microorganismos creditou-se o processo a própria irritação promovida pelo nó, dado o seu volume, pois foram realizados cinco nós quadrados conforme sugerido por Caporn e Roe ${ }^{11}$.

A estabilidade final das técnicas extracapsulares é atribuída ao espessamento da cápsula articular e retináculo, devido à inflamação do procedimento cirúrgico e suturas implantadas ${ }^{7}$. Portanto, o fio utilizado estabilizará a articulação até que ocorra a fibrose periarticular ${ }^{36}$. Dado o resultado final, acredita-se que o objetivo acima foi obtido com os materiais de sutura utilizados, os quais, além de estarem entre aqueles de maior preferência pelos cirurgiões ${ }^{17}$, tem a garantia de esterilidade pelo fabricante, o que não ocorre com o uso por exemplo do fio de náilon leader line $e^{11,16,18,20,25,26}$, e não envolvem o uso de equipamentos especiais e/ou clamp $^{33}$, os quais aumentariam o custo do procedimento.

\section{Conclusões}

A realização da reparação da RLCC pela técnica descrita foi satisfatória perante a entrevista com o proprietário e pelo exame clínico subjetivo realizado pelo veterinário, porém medidas mais objetivas como o 
diâmetro da coxa, volume articular e amplitude articular não evidenciaram total correlação positiva, sugerindo-se a realização de estudos objetivos complementares.

\title{
Agradecimentos
} financeiro.

Agradecimento à FAPESP (Fundação de Amparo a Pesquisa do Estado) pelo auxílio

\section{Retrospective study of extra-articular surgical technique on the repair of cranial cruciate ligament deficency in the dog}

\begin{abstract}
Extra-articular techniques have been widely used on cranial cruciate ligament rupture repair; among these techniques fabelo-tibial suture is one of the most popular. The purpose of the present study was to evaluate the clinical results following at least 4 months of postoperative of cranial cruciate ligament deficiency in 19 dogs (20 joints) using the fabelo-tibial technique. After interviewing the owners a clinical exam was performed by the veterinary and it included gait evaluation, withdraw test and objective measurements of the thigh circumference, stifle angle and stifle diameter of the affected and the contra lateral limbs. The owners evaluated $90 \%$ of the dogs as having an excellent to good recovery, without or with sporadic lameness. The gait and withdraw tests of the clinical evaluation showed satisfactory results. Nevertheless, the objectives measurements don't showed the same results.
\end{abstract}

Key words: Joint. Knee.

\section{Referências}

1 BENNETT, D. et al. A reappraisal of anterior cruciate ligament disease in the dog. J Small Anim Pract, v. 29, n. 5, p. 275-297, 1988

2 LAMPMAN, T. J.; LUND, E. M.; LIPOWITZ, A. J. Cranial cruciate disease: current status od diagnosis, surgery, and risk for disease. Vet Comp Othop Traumatol, v. 3, p. 122-126, 2003.

3 WHITEHAIR, J. G.; VASSEUR, P. B.; WILLITS, N. H. Epidemiology of cranial cruciate ligament rupture in dogs. J Am Vet Med Assoc, v. 203, n. 7, p. 1016-1019, 1993.

4 CHAUVET, A.E. et al. Evaluation of fibular head transposition, lateral fabellar suture, and conservative treatment of cranial cruciate ligament rupture in large dogs: a retrospective study. J Am Anim Hosp Assoc, v. 32, n. 3, p. 247-255, 1996.

5 GAMBARDELLA, P. C.; WALLACE, L. J.; CASSIDY, $F$. Lateral suture technique for management of anterior cruciate ligament rupture in dogs: a retrospective study. J Am Anim Hosp Assoc, v. 17, p. 33-38, 1981.

6 MOORE, K. W.; READ, R. A. Cranial cruciate ligament rupture in the dog. A retrospective study comparing surgical technique. Austr Vet J, v. 72, n. 8, p. 291-285, 1995.

7 PIERMATTEI, D. L.; FLO, G. L. The stifle joint. In: . Brinker, Piermattei, and Flo's handbook of small animal orthopedics and fracture repair. 3. ed. Philadelphia: W. B. Saunders, 1997. p. 516-580.

8 COETZEE, G. L.; LUBEE, A. M. A prospective study comparing two fascial reconstruction techniques to stabilise the cranial cruciate deficient stifle in the dog. Vet Comp Othop Traumatol, v. 8, p. 82-90, 1995.

9 DAVIDSON, J. R. et al. Comparison of stifle biomechanics after two different technique of cranial cruciate ligament repair. Vet Comp Orthop Traumatol, v. 6, n. 1 , p. $172-174,1993$.

10 SHIRES, P. K.; HULSE, D. A.; LIU, W. The underand-over fascial replacement technique for anterior cruciate ligament rupture in dogs: a retrospective study. 
J Am Anim Hosp Assoc, v. 20, p. 69-77, 1982.

11 CAPORN, T. M.; ROE, S. C. Biomechanical evaluation of the suitability of monofilament nylon fishing and leader line for extra-articular stabilization of the canine cruciate-deficient stifle. Vet Comp Othop Traumatol, v. 9, p. 126-133, 1996.

12 DUPUIS, J.; HARARI, J. Cruciate ligament and meniscal injuries in dogs. Comp Cont Educ Pract Vet, v. 15, n. 2 , p. $215-232,1993$

13 FLO, G. Modification of the lateral retinacular imbrication technique for stabilizaing cruciate ligament injuries. J Am Anim Hosp Assoc, v. 11, n. 6, p. 570$575,1975$.

14 HULSE, D. A. et al. Biomechanics of cranial cruciate ligament reconstruction in the dog. I. In Vitro laxity testing. Vet Surg, v. 12, p. 109-112, 1983.

15 PATTERSON, R.H. et al. Biomechanical stability of four cranial cruciate ligament repair techniques in the dog. Vet Surg, v. 20, p. 85-90, 1991.

16 HUBER, D. J.; EGGER, E. L.; JAMES, S. P. The effect of knotting method on the structural properties of large diameter nonabsorbable monofilament sutures. Vet Surg, v. 28, p. 260-267, 1999.

17 KORVICK, D.L.; JOHNSON, A. L.; SCHAEFFER, D. J. Surgeons 'preferences in treating cranial cruciate ligament ruptures in dogs. J Am Vet Med Assoc, v. 205, n. 9, p. 1318-1324, 1994.

18 LEWIS D. D.; MILTHORPE, B. K.; BELLENGER, C. R. Mecahnical comparison of materials used for extracapsular stabilisation of the stifle joint in dogs. Austr Vet J, v. 75, n. 12, p. 890-896, 1997.

19 NWADIKE, B. S.; ROE, S. C. Mechanical comparison of suture material and knot type used for fabelo-tibial sutures. Vet Comp Orthop Traumatol, v.11, p. 47-52, 1998.

20 PEYCKE, L. E. et al. Mechanical comparison of six loop fixation methods with monofolament nylon leader line. Vet Comp Othop Traumatol, v. 4, p. 210-214, 2002.

21 PROSTREDNY, J.M. et al. Effect of suture type on stifle joint biomechanics after extra-articular repair od cranial cruciate ligament transection in the dog. Vet Comp Othop Traumatol, v. 4, p. 144-149, 1991.

22 SELMI, A.L. et al. Centro instantâneo de movimento, na avaliação ex-vivo da reconstrução extra-articular fabelo-tibial, após transecção do ligamento cruzado cranial em cães. Ciência Rural, Santa Maria, v. 33, n. 5 , p. 875-880, 2003.

23 SICARD, G. K.; HAYASHI, K.; MANLEY, P. A Evaluation of 5 types od fishing material, 2 sterilization methods, and a crimp-clamp system for extra-articular stabilization of the canine stifle joint. Vet Surg, v. 31, p. 78-84, 2002.

24 ARNOCZKY, S. P.; MARSHALL, J. L. The cruciate ligaments of the canine stifle: An anatomical and fuctional analysis. Am J Vet Res, v. 38, n. 11, p. 1807$1814,1977$.

25 DULISCH, M. L. Suture reaction following extraarticular stifle stabilization in the dog - Part I: A retrospective study of 161 stifles. J Am Anim Hosp Assoc, v. 17, p. 569-571, 1981.

26 DULISCH, M. L. Suture reaction following extraarticular stifle stabilization in the dog - Part II: A prospective study of 66 stifles. J Am Anim Hosp Assoc, v. 17, p. 572-574, 1981.

27 MCKEE, W. M.; MILLER, A. A self-locking knot for lateral fabellotibial suture stabilization of the cranial cruciate ligament deficient stifle in the dog. Vet Comp Othop Traumatol, v. 12, p. 78-80, 1999.

28 MOREAU, M. DECAMP, C. E. et al.Clinical evaluation of a powder of quality elk velvet antler for the treatment of osteoarthrosis in dogs. Can Vet J, v. 45, n. 2, p. 133139, 2004.

29 WHITNEY, W. O. Arthroscopically assisted surgery of the stifle joint. In: BEALE, B. S.; HULSE, D. A.; SCHULZ, K. S.; WHITNEY, W. O. Small animal arthroscopy. Philadelphia: W. B. Saunders, 2003. p. 116-157.

30 PIERMATTEI, D. L.; JOHNSON, K. A. The Hindlimb. In:_. An atlas of surgical approaches to the bones and joints of the dog and cat. 4. ed. Philadelphia: W. B. Saunders, 2004. p. 342 .

31 VASSEUR, P.B. Clinical results following nonoperative management for rupture of the cranial cruciate ligament in dogs. Vet Surg, v. 13, n. 4, p. 243246, 1984.

32 FLO, G.; DE YOUNG, D. Meniscal injuries and medial meniscectomy in the canine stifle. J Am Anim Hosp Assoc, v. 14, n. 8, p. 683-689, 1978.

33 RALPHS, S. C.; WHITNEY, W. O. Arthroscopic evaluation of menisci in dogs with cranial cruciate ligament injuries: 100 cases (1999-2000). J Am Vet Med Assoc, v. 221, n. 11, p. 1601-1604, 2002.

34 BALLAGAS, A.J.; MONHENDERSON, R.A.; GILLETTE, R. Pre- and posoperative force plate analysis of dogs with experimentally transected cranial cruciate ligaments treated using tibial plateau leveling osteotomy. Vet Surg, v. 33, n. 5, p. 187-190, 2004.

35 ANDERSON, C.C. et al. Biomechanical evaluation of a crimp clamp system for loop fixation of monofilament nylon leader material used for stabilization of the canine stifle joint. Vet Surg, v. 27, p. 533-539, 1998

36 DECAMP, C. E. et al. Kinematic evaluation of gait in dogs with cranial cruciate ligament rupture. Am J Vet Res, v. 57, n. 1, p. 120-126, 1996. 